\title{
IDENTIFICAÇÃO NÃO-LINEAR CAIXA-CINZA: UMA REVISÃO E NOVOS RESULTADOS
}

\author{
Marcelo V. Corrêa* \\ mcorrea@unilestemg.br
}

\author{
Luis A. Aguirre ${ }^{\dagger}$ \\ aguirre@cpdee.ufmg.br
}

${ }^{*}$ Curso de Engenharia Elétrica, Centro Universitário do Leste de Minas Gerais-Unileste-MG Av. Tancredo Neves 3500, Cel. Fabriciano, MG, Brazil.

${ }^{\dagger}$ Departamento de Engenharia Eletrônica, Universidade Federal de Minas Gerais Av. Antônio Carlos 6627, 31270-901 Belo Horizonte, MG, Brazil.

\begin{abstract}
This paper presents a review of recent developments about gray-box identification. Different procedures are grouped based on how they handle auxiliary information in the identification process. Properties of NARMAX (nonlinear autoregressive moving average model with exogenous inputs) polynomial models applied in gray-box identification are presented. This paper discusses how to use auxiliary information about the process static function in order to aid in the model structure selection and parameter estimation. Such ideas are illustrated by means of two numerical examples, one simulated and one that uses real data. In both examples black-box and gray-box models are obtained and compared in order to highlight the main features of gray-box identification.
\end{abstract}

KEYWORDS: Non-linear identification, gray-box, NARMAX models, auxiliar information.

\section{RESUMO}

Este artigo apresenta uma breve revisão da literatura enfocando recentes desenvolvimentos na área de identificação caixa-cinza. Os diversos métodos são agrupados

Artigo submetido em $15 / 08 / 02$

1a. Revisão em 06/12/02; 2a. Revisão em 01/03/03

Aceito sob recomendação do Ed. Assoc. Prof. José R. C. Piqueira dependendo de como a informação auxiliar é utilizada no processo de identificação. Discutem-se propriedades da representação NARMAX (nonlinear autoregressive moving average model with exogenous inputs) polinomial aplicando-se identificação caixa-cinza. Mecanismos de utilização de informação auxiliar na seleção de estrutura e na estimação de parâmetros de modelos NARMAX polinomiais são discutidos e ilustrados usando dois exemplos numéricos, um simulado e outro com dados medidos. Em ambos os casos obtêm-se modelos via procedimentos caixa-preta e caixa-cinza. As características dos modelos assim obtidos são comparadas de forma a ilustrar aspectos importantes da identificação caixa-cinza.

PALAVRAS-CHAVE: Identificação não-linear, caixacinza, modelos NARMAX, informação auxiliar.

\section{INTRODUÇÃO}

Em métodos de identificação caixa-preta, nenhuma informação sobre o sistema está disponível além dos dados ou, se disponível, não é usada no procedimento de obtenção do modelo. Neste caso, apenas dados de entrada e saída do sistema são usados durante a identificação. A escolha da representação e da sua estrutura é feita de forma empírica nos casos mais simples. Em casos mais complexos, como por exemplo na identificação de sistemas não-lineares, esta escolha é crítica o que justifica o uso de métodos mais sofisticados para seleção da estru- 


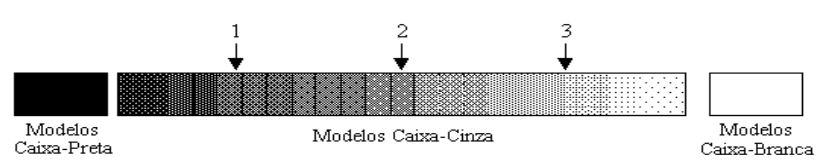

Figura 1: Escala cinzenta definindo os diferentes níveis de informação auxiliar na identificação caixa-cinza (Karplus, 1976). Por exemplo: procedimentos em que o uso de informação auxiliar se limita à definição de termos candidatos, no caso de modelos NARMAX, ou da função de ativação, no caso de redes neurais, se situariam na região 1; procedimentos em que se usa informação auxiliar na estimação de parâmetros se situam na região 2; nos casos em que a estrutura é definida por leis físicas e parâmetros estimados com dados de entrada e saída, região 3 .

tura do modelo. Tais métodos baseiam-se em técnicas de álgebra linear e em conceitos de estatística. Para estimação de parâmetros utilizam-se procedimentos de otimização sem restrições. Na identificação caixa-preta não existe nenhuma relação óbvia entre a estrutura e seus parâmetros com aspectos físicos do sistema sendo identificado.

$\mathrm{Na}$ identificação caixa-branca ou simplesmente modelagem física, o processo de obtenção do modelo se baseia em leis e princípios físicos. Todos os parâmetros são conhecidos, ou previamente determinados. Dados de entrada e saída do sistema, quando disponíveis são usados apenas para validar o modelo. $\mathrm{Na}$ identificação caixa branca, todos os termos da estrutura, e seus parâmetros, possuem significado físico (Garcia, 1997).

Como desvantagens da identificação caixa-preta podese citar, em geral, o fato de a estrutura do modelo não possuir significado físico, a dificuldade para sua seleção e, em muitos casos, o número excessivo de parâmetros. Como vantagens, em geral, são enumeradas a relativa facilidade de obtenção e a possibilidade de se escolher estruturas mais adequadas para o projeto de sistemas de controle (Pottmann e Pearson, 1998).

Como desvantagem da modelagem caixa-branca, destaca-se a dificuldade de obtenção do modelo. Em geral, as equações físicas envolvidas em um processo, assim como seus parâmetros, não são totalmente conhecidas. Muitas vezes, as relações são por demais complexas e não podem ser determinadas. Como principal vantagem, destaca-se o significado físico do modelo obtido.

Essas duas formas de modelagem podem ser interpreta- das como os dois extremos de técnicas de modelagem. A rigor, qualquer procedimento que não esteja em nenhum desses extremos pode ser denominada de identificação caixa-cinza. Esta área de conhecimento busca combinar as vantagens dos procedimentos de identificação caixa-preta e caixa-branca. Nesse caso, tanto dados de entrada e saída obtidos no sistema, quanto informação auxiliar são usados na identificação. A identificação caixa-cinza é uma área bastante ampla e por se tratar de um assunto relativamente novo, muitos problemas estão praticamente em aberto. O tipo de conhecimento auxiliar utilizado, assim como o nível de conhecimento, varia de caso para caso. A Figura 1 mostra que a tonalidade do "cinza" varia de acordo com o nível e a quantidade de informação auxiliar usada no processo de modelagem (Karplus, 1976; Sohlberg, 1998).

Um termo muito utilizado na literatura é conhecimento a priori. Apesar de não haver uma definição formal para tal conhecimento, parece adequado chamar de conhecimento a priori "apenas" informação oriunda das leis físicas que regem o processo e qualquer outra informação, ainda que esteja disponível antes de se obter o modelo final será chamada informação auxiliar (Neponuceno, 2002).

Vale ressaltar que, na identificação caixa-branca, desvantagens também podem ser enumeradas. Por exemplo, os modelos caixa-cinza, à semelhança dos caixapreta, têm menos significado físico que os caixa-branca. Note que, se a informação auxiliar disponível for incorreta, seu uso na identificação caixa-cinza será conflituoso com os dados, podendo resultar em um modelo de baixa qualidade. Tal não ocorre na identificação caixa-preta.

Uma síntese das vantagens de cada abordagem de identificação, além das dificuldades que serviram como motivação para o desenvolvimento de novas técnicas pode ser bem visualizada através da Figura 2 (Aguirre, 2000b). Como exemplo pode ser observado que dificuldades do "Modelo Matemático Não-Linear Geral" é a complexidade de sua estrutura e a dificuldade para extrair informação do modelo. A questão da seleção da estrutura de modelos não-lineares gerais deu origem ao desenvolvimento de algoritmos para sua seleção. Técnicas de identificação caixa-cinza podem ser interpretadas como contribuições no sentido de melhorar a escolha de estrutura e a estimação de parâmetros pelo uso de informação auxiliar.

De uma maneira geral, os modelos caixa-preta são obtidos com maior facilidade. O sucesso de tais modelos depende fortemente da qualidade dos dados (dinâmicos) usados. Pode-se dizer que bons modelos caixa-preta só 


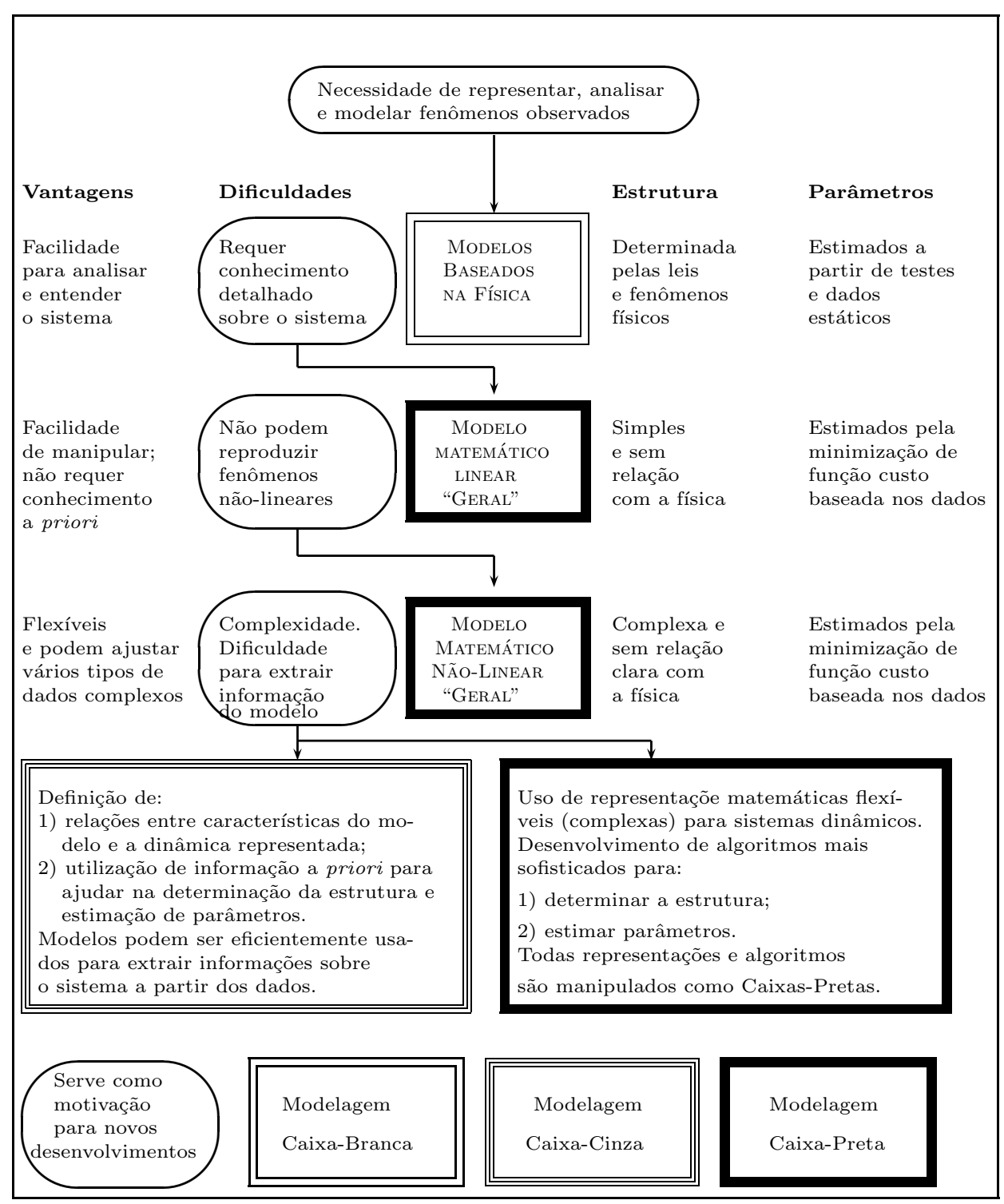

Figura 2: Representação pictórica das dificuldades de cada classe de modelos e das motivações para o desenvolvimento de novos procedimentos (Aguirre, 2000b).

são possíveis se as séries temporais usadas (dados dinâmicos) contiverem toda a informação a respeito do sistema que se deseja modelar. Na maioria das situações a obtençaõ de tais dados é muito custosa e nem sempre possível. Por outro lado, em muitos casos, informação auxiliar está disponível (Simutis et al., 1997) e seu uso no processo de identificação contribui significativamente para a melhora do modelo obtido (Murankami e Seborg, 2000; Abony et al., 2001). A identificação caixacinza, como será visto, permite obter modelos mais globais mesmo com dados limitados a certa região de operação (Funkquist, 1997).

Assim como as redes neurais e as RBF's (radial basis functions), a representação NARMAX polinomial é tipicamente caixa-preta. Porém, esta última, devido a características algébricas (Corrêa, 2001; Aguirre et al., 2002), apresenta relativa facilidade de se incorporar informação auxiliar tanto na fase de seleção de estrutura (Aguirre et al., 2000) quanto na estimação de parâmetros (Corrêa et al., 2002). Esta representação, que já vem sendo largamente utilizada na identificação caixa-preta, tem se mostrado bastante eficiente em identificação caixa-cinza. Muitas das idéias propostas para esta representação são adaptáveis a outras representações (Amaral, 2001).

Este artigo apresenta uma breve revisão sobre recentes desenvolvimentos na área de identificação caixa-cinza. Além disso, resultados de identificação caixa-cinza uti- 
lizando a representação NARMAX polinomial são apresentados. Para tal, este artigo é organizado como se segue. Na seção 2 apresenta-se uma revisão bibliográfica. Na seção 3 algumas propriedades da representação NARMAX polinomial são discutidas no contexto de identificação caixa-cinza. A seguir, na seção 4, descrevese como usar informação auxiliar em modelos NARMAX polinomiais. Na seção 5 modelos obtidos via identificação caixa-cinza são comparados com a identificação caixa-preta. Por último, as principais conclusões do estudo são apresentadas.

\section{ESTADO DA ARTE}

Nesta seção apresenta-se como o problema de identificação caixa-cinza tem sido tratado na literatura recente. Para tal os procedimentos são agrupados em: (i) procedimentos aplicados a modelos baseados em redes neurais; (ii) identificação caixa-cinza física, que se baseia na determinação da estrutura dos modelos pelas leis físicas do processo, ou seja, seguindo a convenção feita, neste caso a informação auxiliar é informação a priori; (iii) caixa-cinza semifísica, que utilizam conhecimento auxiliar na definição da estrutura dos modelos, porém, sem necessariamente utilizar equações obtidas a partir de leis físicas; (iv) identificação caixa-cinza, que apresenta trabalhos que não podem ser agrupados nas categorias anteriores. Por último são apresentados procedimentos para uso de informação auxiliar na seleção de estrutura de modelos NARMAX polinomiais.

\subsection{Uso de Conhecimento Auxiliar em Re- des Neurais}

O uso de conhecimento auxiliar em redes neurais foi tratado em (Joerding e Meador, 1991; Thompson e Kramer, 1994; Cubillos et al., 1996; Amaral, 2001). Thompson e Kramer defendem a utilização de informação auxiliar, principalmente em situações em que os dados são compostos por uma série temporal de curta duração e seriamente contaminada por ruído. O tipo de conhecimento auxiliar utilizado é (i) monotonicidade e assintoticidade, (ii) o propósito de modelagem, (iii) restrições "fortes" baseadas em princípios físicos e (iv) desempenho de modelos previamente obtidos.

Como forma de utilizar tais informações os autores citam: (i) a definição da estrutura e (ii) a forma de estimação de parâmetros. Tal procedimento reduz a dimensão do espaço de parâmetros e a quantidade de dados necessários para sua estimação.

Cada tipo de conhecimento auxiliar tem um papel dife- rente durante a identificação, que depende dos seguintes atributos: (i) tipo, se a relação é de igualdade ou desigualdade; (ii) forma, se a estrutura funcional da relação é conhecida ou não; (iii) precedência, a precedência em relação aos dados; e (iv) variáveis, quais variáveis são envolvidas. O Exemplo 2.1 permite um entendimento melhor de como a informação auxiliar é incorporada na seleção de estrutura (Thompson e Kramer, 1994).

Exemplo 2.1 Considere um sistema de mistura de gás não ideal com componentes $A, B$, e $C$. Usando dados experimentais, deseja-se determinar a relação entre volume $V$, pressão $P$, temperatura $T$, total de moles $n$ e composição (fração molar) $X=\left[\begin{array}{lll}X_{A} & X_{B} & X_{C}\end{array}\right]^{T}$.

Considere ainda a Tabela 1 que apresenta cada informação auxiliar disponível, seus atributos e como utilizá-la na definição da estrutura da rede. Como exemplo podese notar que: na linha 1 , a informação de que o volume $V$ é função das variáveis $P, T, n$ e $X$, mas sua forma é desconhecida, motivou a inclusão no modelo de uma rede neural. Na linha 2, como existe uma equação de forma conhecida, então no modelo é selecionado um préprocessador paramétrico. O mesmo deve ser feito para as demais informações disponíveis.

No Exemplo 2.1 observa-se a utilização de informação auxiliar na definição de estruturas híbridas, em geral combinando modelos paramétricos fixos com redes neurais, em série ou paralelo. A utilização de estruturas híbridas para identificação de sistemas dinâmicos pode também ser visto em (Cubillos et al., 1996; Cubillos e Lima, 1997).

Em (Joerding e Meador, 1991) três benefícios do uso de informação auxiliar em redes neurais são citados: (i) previne que a rede faça predições impossíveis ou não plausíveis; (ii) melhora a capacidade de generalização e (iii) aumenta a eficiência do treinamento. Os autores propõem a utilização de informação auxiliar na definição da arquitetura da rede neural e no estabelecimento de restrições na estimação dos pesos. Outros resultados do uso de informação auxiliar em redes neurais também podem ser vistos em (Amaral, 2001).

\subsection{Modelagem Caixa-Cinza Física}

Dentre os trabalhos que tratam da identificação caixacinza, destaca-se o grande número de referências aos trabalhos de Bohlin (Bohlin, 1994; Bohlin e Graebe, 1995). Nestes, Bohlin e colegas apresentam um procedimento que denominam como modelagem semifísica probabilística. 
Tabela 1: Exemplo de uso de conhecimento auxiliar no modelo de uma mistura de gás (Thompson e Kramer, 1994)

\begin{tabular}{|c|c|c|c|c|}
\hline & Relação & \multicolumn{2}{|c|}{ Atributos } & Estrutura \\
\hline 1. & $V=f(P, T, n, X)$ & $\begin{array}{l}\text { Tipo: } \\
\text { Forma: } \\
\text { Precedência: } \\
\text { Variáveis: }\end{array}$ & $\begin{array}{l}\text { igualdade } \\
\text { desconhecida } \\
\text { grande } \\
\text { entrada \& saída }\end{array}$ & $\begin{array}{c}\text { rede } \\
\text { neural }\end{array}$ \\
\hline 2. & $\begin{array}{c}\text { soma das frações } \\
\text { molares } \\
\sum X_{i}=1\end{array}$ & $\begin{array}{l}\text { Tipo: } \\
\text { Forma: } \\
\text { Precedência: } \\
\text { Variáveis: }\end{array}$ & $\begin{array}{l}\text { igualdade } \\
\text { conhecida } \\
\text { grande } \\
\text { entradas }\end{array}$ & $\begin{array}{c}\text { pré- } \\
\text { processador } \\
\text { paramétrico }\end{array}$ \\
\hline 3. & $\begin{array}{c}\text { Entradas } \\
\text { não-negativas } \\
x \geq 0\end{array}$ & $\begin{array}{l}\text { Tipo: } \\
\text { Forma } \\
\text { Precedência: } \\
\text { Variáveis: }\end{array}$ & $\begin{array}{l}\text { desigualdade } \\
\text { conhecida } \\
\text { grande } \\
\text { entradas }\end{array}$ & $\begin{array}{c}\text { pré- } \\
\text { processador } \\
\text { paramétrico }\end{array}$ \\
\hline 4 . & $\begin{array}{c}\text { Saídas } \\
\text { não-negativas } \\
y(x) \geq 0 \\
\text { para todo } x\end{array}$ & $\begin{array}{l}\text { Tipo } \\
\text { Forma: } \\
\text { Precedência: } \\
\text { Variáveis: }\end{array}$ & $\begin{array}{l}\text { desigualdade } \\
\text { conhecida } \\
\text { grande } \\
\text { entradas }\end{array}$ & $\begin{array}{c}\text { modelo } \\
\text { paramétrico } \\
\text { na saída }\end{array}$ \\
\hline
\end{tabular}

Em (Bohlin e Graebe, 1995) o usuário especifica uma estrutura inicial para o modelo, e o software implementa a estimação de parâmetros e a validação. As etapas do procedimento de modelagem são apresentadas via um fluxograma reproduzido na Figura 3. O usuário interage com o computador em uma dupla malha de refinamento e refutação de estruturas selecionadas, e deve parar a modelagem quando a melhor alternativa de uma determinada estrutura servir aos seus propósitos. A parte realizada pelo computador se resume a estimar os parâmetros e validar o modelo. Esta se dá tanto por procedimentos estatísticos quanto por simulação dinâmica.

A definição da estrutura inicial, assim como das futuras modificações, baseia-se no conhecimento de leis físicas que regem o processo. Uma detalhada discussão desse procedimento com aplicações industriais pode ser vista em (Sohlberg, 1998).

Modelos qualitativos foram propostos como uma estrutura unificada para modelagem caixa-cinza com diferentes níveis de precisão (Jorgensen e Hangos, 1995). Tais modelos são compostos de equações algébricas diferenciais qualitativas (QDAEs do inglês Qualitative Diferencial Algebraic Equations), no caso contínuo, e equações algébricas de diferenças qualitativas (QDDAEs), para o caso discreto. O espaço de intervalos das variáveis e parâmetros em um modelo QDAE é definido como um conjunto de intervalos de números/símbolos fixos atribuídos individualmente para cada variável real. As dependências parcialmente conhecidas são descritas por pares de funções denominadas funções envelope.
Em (Sadegh et al., 1995) é apresentado um procedimento para projeto de sinal de excitação que maximiza o valor da informação auxiliar disponível. O problema é tratado como um caso de otimização, sendo o objetivo determinar uma entrada ótima para estimação de determinados parâmetros.

\subsection{Modelagem Caixa-Cinza Semifísica}

$\mathrm{Na}$ identificação caixa-cinza semifísica, conhecimento baseado em leis físicas é usado para sugerir combinações não-lineares entre os sinais medidos (Lindskog e Ljung, 1995).

Em (Kárný et al., 1995) é proposto um procedimento, que segundo os autores, fornece (i) uma nova ferramenta tanto para modelagem caixa-preta quanto para caixacinza, (ii) uma inédita adaptação de modelos probabilísticos e (iii) uma forma de reduzir a complexidade do modelo.

O procedimento é aplicado a um modelo ARX, ilustrando a aplicação nas seguintes tarefas: (i) escolha da estrutura apropriada; (ii) incorporação de informação auxiliar nas condições iniciais do algoritmo de mínimos quadrados recursivos; (iii) construção de referência para uma técnica de esquecimento avançada; (iv) aproximação de um modelo analítico complexo por um modelo ARX. 


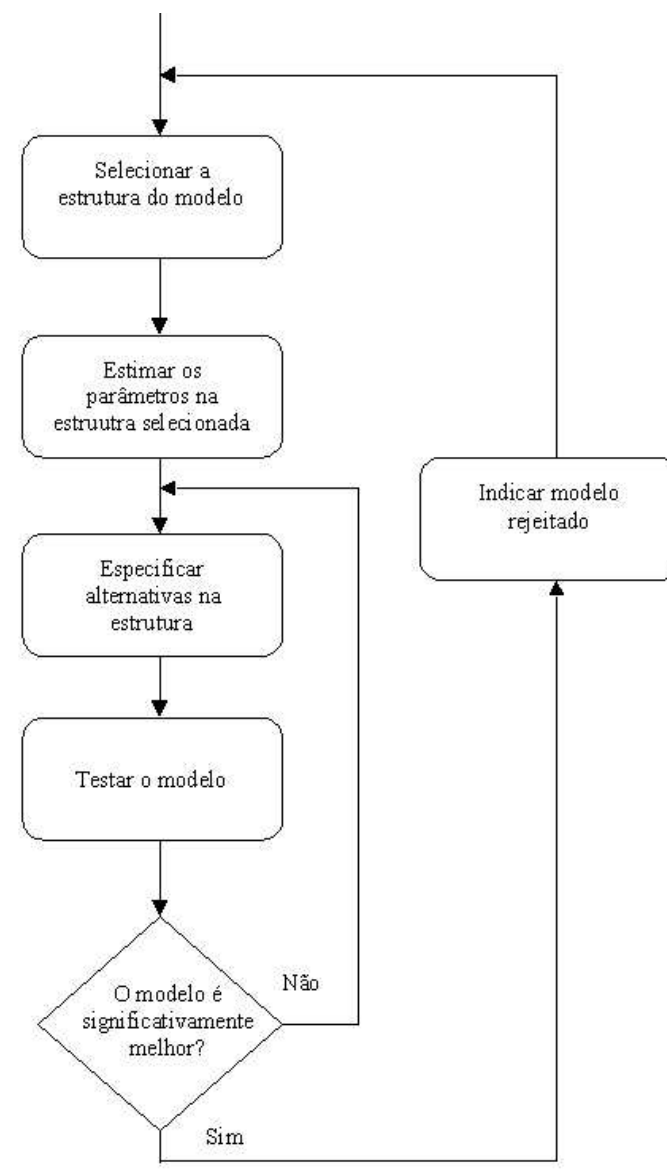

Figura 3: Fluxograma ilustrativo do procedimento interativo de refinamento de estrutura (Bohlin e Graebe, 1995).

\subsection{Identificação Caixa-Cinza}

Nesta seção será apresentada síntese de trabalhos em que a informação auxiliar utilizada não é necessariamente obtida a partir de leis físicas. Os trabalhos aqui apresentados partem de informações a respeito da curva estática. A utilização desse tipo de informação se justifica pois relações em estado estacionário são mais fáceis de serem obtidas e eventualmente podem até estar previamente disponíveis.

Entre os trabalhos que propõem o uso da curva estática de sistemas dinâmicos, destaca-se o apresentado por Pottmann e Pearson (1998). O objetivo de modelagem é a obtenção de modelos NARMAX que reproduzam exatamente as características estacionárias conhecidas do sistema.

Utilizam uma representação geral denominada $N A R$ $M A X$ de blocos interconectados, tendo como casos par-

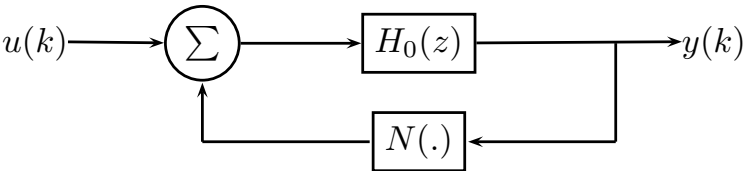

Figura 4: Modelo de blocos orientados com realimentação, estrutura I (Pottmann e Pearson, 1998).

ticulares as representações de Wiener e Hammerstein (Wiener, 1958; Pearson e Pottmann, 2000). Quatro estruturas básicas para modelagem são apresentadas.

A estrutura I, apresentada na Figura 4, consiste em um bloco contendo um elemento dinâmico linear no ramo direto, e outro, com característica estática não-linear na realimentação. A informação da curva estática do sistema é utilizada na definição da função estática nãolinear, $N($.$) .$

Outras estruturas apresentadas pelos autores se baseiam no diagrama em blocos representado na Figura 5 e na sua variação, Figura 6 , sendo que esta última é obtida através da aplicação de regras algébricas de manipulação de diagramas em blocos.

Outros trabalhos que utilizam informação auxiliar na seleção de estrutura de modelos podem ser vistos em (Petrick e Wigdorowitz, 1997; Aguirre et al., 2000). Petrick e colega citam que poucas ferramentas existem para incorporar informação auxiliar na seleção de estrutura de modelos não-lineares. Apresentam uma abordagem para utilização de técnicas de análise de sistemas não-lineares na seleção de estrutura. Mostram a possibilidade de se utilizar resultados do cálculo dos expoentes de Lyapunov, mapas de Poincaré e dimensão de correlação.

Como exemplo, aplicam a técnica em dados obtidos no circuito de Chua. Através do retrato de fase do atrator reconstruído, mostram que é possível inferir o número e a posição aproximada de cada ponto fixo. O mapa de Poincaré é usado para inferir o comportamento do sistema próximo aos pontos fixos. Com as informações extraídas, procura-se uma estrutura não-linear mínima capaz de reproduzir tais características observadas nos dados. 


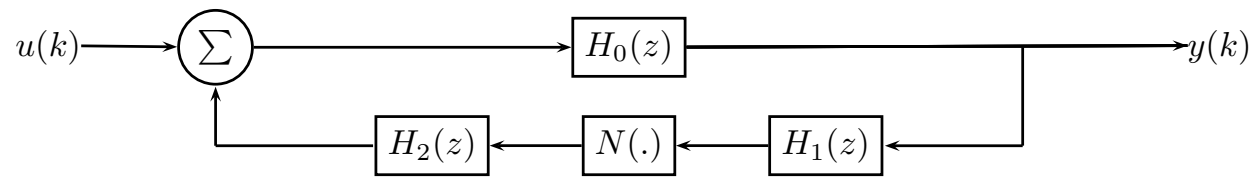

Figura 5: Modelo de Blocos Orientados com Realimentação, estrutura IV (Pottmann e Pearson, 1998).

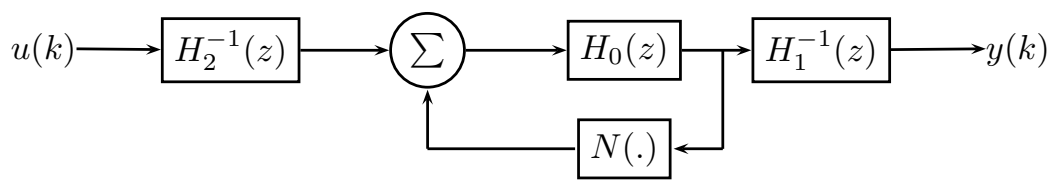

Figura 6: Modelo de Blocos Orientados com Realimentação. Variação da estrutura IV, obtida por manipulações algébricas (Pottmann e Pearson, 1998).

\subsubsection{Uso de Informação auxiliar no estimador de parâmetros}

Outro alvo buscado na identificação caixa-cinza é o uso de informação auxiliar na estimação de parâmetros (Bai e Sastry, 1986; Tulleken, 1993; Johansen, 1996). O objetivo é garantir que o modelo obtido reproduza fielmente características previamente conhecidas.

Em (Bai e Sastry, 1986) é considerado o problema de estimar ganho de uma função de transferência para um sistema SISO. A técnica apresentada é aplicada a um sistema simulado.

Tulleken (1993) discute o problema de uso de informação auxiliar ao estimar parâmetros usando modelos ARMAX e discute a utilização de informação sobre estabilidade e ganho estacionário na forma de restrições nos parâmetros.

Tal procedimento parece ser atraente em condições experimentais não-ideais. Na maioria dos casos, ou mesmo na sua totalidade, as condições ideais necessárias para uma identificação não estão presentes em situações reais. Nesses casos o autor defende a utilização de informação auxiliar ao estimar parâmetros. Como conhecimento de "vital" importância em um processo de modelagem, o autor cita as "regiões admissíveis para localização de pólos" e "ganhos estáticos".

Tulleken (1993) considera modelos do tipo ARX. Com relação à localização dos pólos considera os seguintes tipos de informação auxiliar: (i) estabilidade do sistema em malha aberta; (ii) freqüência de amostragem correta e (iii) coeficiente de amortecimento previamente conhecido.

Para cada um dos itens citados acima, o autor apresenta restrições a serem incorporadas no estimador de parâmetros de um modelo ARX. A estabilidade do modelo ARX em malha aberta é obtida restringindo a localização dos pólos dentro do círculo de raio unitário no plano complexo $\mathbb{C}$. No caso da freqüência de amostragem, o autor parte do princípio que, se o sistema está corretamente amostrado, então seus pólos não podem estar situados no semiplano negativo de $\mathbb{C}$. Procedimento equivalente é apresentado para o caso de coeficiente de amortecimento conhecido.

Com relação ao ganho estático, o autor cita que: “...'bons' modelos geralmente são pobres na aproximação de características de baixa freqüência". Ressalta que o uso de informação sobre o ganho estático introduz um significativo aumento da qualidade do modelo em geral, o que pode ser crucial para um projeto de controle satisfatório (Tulleken, 1993). Um procedimento parecido é apresentado por (Murankami e Seborg, 2000) em que os parâmetros de modelos ARX são estimados de forma a garantir que o modelo final tenha um ganho pré-especificado, que é a informação auxiliar utilizada.

Conhecimento auxiliar pode ser incorporado no estimador de parâmetros através de restrições e penalidades na função objetivo (Johansen, 1996). Mostra-se uma aplicação com modelos NARMAX, cuja não-linearidade é implementada através de uma rede neural. Como exem- 
plos possíveis de serem incorporados, o autor cita: (i) modelo padrão, (ii) modelo linear de ruído, (iii) modelo linear conhecido, (iv) balanço de massa em estado estacionário e (v) estabilidade.

Como aspectos negativos da metodologia proposta, o autor cita a complexidade computacional e a dificuldade de interpretar e analisar o modelo obtido, como principal vantagem, a flexibilidade do método. Essa flexibilidade se refere à possibilidade de incorporação de conhecimento auxiliar de várias formas. Vale ressaltar que o autor cita que "Mais trabalhos são necessários para desenvolver procedimentos práticos de engenharia para codificar conhecimentos a priori".

\subsection{Uso de Informação Auxiliar na Sele- ção de Estrutura de Modelos NAR- MAX Polinomiais}

O procedimento apresentado nesta seção difere dos anteriores, pois a incorporação da informação auxiliar se baseia fundamentalmente em um estudo aprofundado das características da representação. A partir desse estudo determina-se como deve ocorrer o uso da informação auxiliar.

A base desse desenvolvimento são os conceitos de agrupamento de termos e coeficiente de agrupamento apresentados em (Aguirre e Billings, 1995). A partir desses conceitos, em (Aguirre e Mendes, 1996) são deduzidas relações entre agrupamento de termos e o número e a localização de pontos fixos. Com estas relações, a partir de informação auxiliar sobre o número e localização dos pontos fixos do sistema a ser modelado selecionam-se os agrupamentos de termos candidatos ao modelo. A escolha dos termos do modelo precisa ser feita por algum método disponível, como por exemplo usando-se o critério ERR (do inglês error reduction ratio) (Korenberg et al., 1988; Billings et al., 1989).

Dentro da escala "cinza" apresentada na Figura 1, esse procedimento pode ser classificado como um "cinzaescuro", porque o grau de utilização da informação auxiliar é restrito. A partir da seleção dos agrupamentos candidatos o procedimento é puramente caixa-preta, tendo sido aplicado com sucesso na identificação de diversos sistemas autônomos caóticos, entre esses destacase a identificação a partir de dados reais em um oscilador não-linear (Aguirre et al., 1997).

Esse procedimento foi estendido para identificação de sistemas não-autonômos em (Aguirre et al., 2002). O objetivo é possibilitar selecionar a estrutura da representação a partir de informação de ganho em estado es- tacionário. Fundamenta-se, da mesma forma que para o caso autômono, no estudo de como é mapeada no modelo a função de ganho de estado estacionário. A partir desse estudo e da informação auxiliar disponível, são escolhidos os agrupamentos de termos candidatos.

\section{PROPRIEDADES DAS REPRESENTA- COÕES NARMAX POLINOMIAIS APLI- CADAS À IDENTIFICAÇÃO CAIXA- CINZA}

Um modelo NARMAX polinomial é representado por (Leontaritis e Billings, 1985):

$$
\begin{gathered}
y(k)=F^{\ell}\left[y(k-1), y(k-2), \cdots, y\left(k-n_{y}\right),\right. \\
u(k-1), \cdots, \quad u\left(k-n_{u}\right), \\
\\
\left.e(k-1), \cdots, e\left(k-n_{e}\right)\right]+e(k),
\end{gathered}
$$

sendo que $u(k)$ e $y(k)$ representam, respectivamente, a entrada e a saída nos instantes discretos $k(k=1,2, \cdots)$, $e(k)$ representa um ruído independente não-observável, $n_{u}, n_{y}$ e $n_{e}$ são, respectivamente, os máximos atrasos dos termos da entrada, saída e ruído. $F^{\ell}$ é uma função polinomial não-linear com grau de não-linearidade $\ell$. Antes de prosseguir, chama-se a atenção para o fato de que para reduzir o efeito da polarização durante a estimação de parâmetros, modelos NARMAX serão estimados utilizando-se o estimador estendido de mínimos quadrados (Aguirre, 2000b), mas apenas a parte determinística, ou seja, a parte NARX será utilizada na análise e simulação.

\subsection{Autovalores de Modelos de Ordem Su- perior}

O procedimento adotado para obter a função autovalores de modelos NARX polinomiais é: (i) representar o modelo na forma de equação de estados, linearizando-o em torno dos pontos fixos através da matriz jacobiana, (ii) a partir dessa matriz montar a equação característica, determinando seus autovalores em função do ponto de operação e, (iii) mapear os autovalores do modelo discreto, plano $z$, no plano $s$. Para isso serão usados novos conceitos de agrupamento de termos, denominados agrupamentos- $d$ e coeficientes- $d$. Estes conceitos são apresentados a seguir.

Definição 3.1 (Corrêa, 2001) Termos na forma $y(k-$ d) $)^{p} u(k-j)^{m}$ para $m=0, \cdots, \ell, p=0, \cdots, \ell \operatorname{com} m+$ $p \leq \ell$ e $j=1, \cdots, n_{u}$ são chamados agrupamentos- $d$ e são representados por $\Omega_{y_{d}^{p} u^{m}}$. A soma de todos os coeficientes de um agrupamento de termos de ordem $d$ 
são chamados de coeficientes-d e são representados por

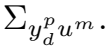

Vale ressaltar que

$$
\begin{aligned}
\Omega_{y} & =\Omega_{y_{1}} \cup \Omega_{y_{2}} \cup \Omega_{y_{3}} \ldots \\
\Omega_{y^{2}} & =\Omega_{y_{1} y_{2}} \cup \Omega_{y_{1}^{2}} \cup \Omega_{y_{2}^{2}} \ldots \\
& \vdots \\
\Sigma_{y} & =\sum_{i=1}^{n_{y}} \Sigma_{y_{i}}
\end{aligned}
$$

o que significa que no caso de modelos de primeira ordem os agrupamentos de termos são iguais aos agrupamentosd. O mesmo ocorre com os coeficientes de agrupamentos.

Para deduzir a função de autovalores, o modelo NARX polinomial será representado no espaço de estados linearizando-o em torno de cada estado estacionário. Chamando $\mathbf{Y}(k-1)$ de vetor de estado, a equação (1) pode ser escrita na forma

$$
\left[\begin{array}{c}
y\left(k-n_{y}+1\right) \\
y\left(k-n_{y}+2\right) \\
\vdots \\
y(k-1) \\
y(k)
\end{array}\right]=\mathbf{D}_{\mathbf{f}}\left[\begin{array}{c}
y\left(k-n_{y}\right) \\
y\left(k-n_{y}+1\right) \\
\vdots \\
y(k-2) \\
y(k-1)
\end{array}\right]+\mathbf{B U}(k)
$$

sendo

$$
\begin{gathered}
\mathbf{D}_{\mathbf{f}}=\left[\begin{array}{ccccc}
0 & 1 & \cdots & 0 & 0 \\
0 & 0 & \cdots & 0 & 0 \\
\vdots & \vdots & \cdots & \vdots & \vdots \\
0 & 0 & \cdots & 0 & 1 \\
\triangle_{d} & \triangle_{d-1} & \cdots & \triangle_{2} & \triangle_{1}
\end{array}\right] \\
\triangle_{d}=\left.\frac{\partial y(k)}{\partial y(k-d)}\right|_{\bar{y}, \bar{u}},
\end{gathered}
$$

e $d$ é o atraso da saída $y$. Nota-se que a expressão de $\triangle_{d}$ é determinada em função da derivada parcial, avaliada para $y=\bar{y}$ e $u=\bar{u}$, ou seja, no ponto de operação. Com objetivo de se obter uma expressão geral para $\triangle_{d}$ serão usados os conceitos de agrupamentos- $d$ e coeficientes- $d$. Assim, a equação (5) pode ser escrita

$$
\begin{aligned}
\triangle_{d}(\bar{y}, \bar{u})=\Sigma_{y_{d}}+\sum_{p=2}^{\ell} p \Sigma_{y_{d}^{p}} \bar{y}^{p-1} & \\
& \quad+\sum_{m=1}^{\ell} \sum_{p=1}^{\ell-m} p \Sigma_{y_{d}^{p} u^{m}} \bar{y}^{p-1} \bar{u}^{m},
\end{aligned}
$$

ou, escrita como:

$$
\triangle_{d}\left(\bar{x}_{\triangle_{d_{i}}}\right)=\triangle_{d_{0}}+\sum_{i=1}^{n \triangle_{d}} \triangle_{d_{i}} \bar{x}_{\triangle_{d_{i}}}
$$

sendo $\triangle_{d_{0}}=\Sigma_{y d}, \triangle_{d_{1,2, \ldots, d}}$ constantes que dependem dos coeficientes- $d$, e $\bar{x}_{\triangle_{d_{i}}}$ são sinais dos quais a variável $\triangle_{d}$ depende. Estes sinais aparecem nos modelos NARX polinomiais como produto de $y(k-d)$.

Os autovalores do modelo NARX são as soluções de $\operatorname{det}\left|\lambda I-\mathbf{D}_{\mathbf{f}}\right|=0$, sendo $I$ uma matriz identidade de dimensão adequada. Então, a equação característica será dada por

$$
\lambda^{d}-\triangle_{1} \lambda^{d-1}-\ldots-\triangle_{d-1} \lambda-\triangle_{d}=0
$$

sendo as raízes da equação (8) os autovalores $\lambda$ do modelo NARX polinomial no ponto de operação $(\bar{u}, \bar{y})$.

\subsection{Ganho em Estado Estacionário}

Como o objetivo é o de determinar o ganho estático $K_{\mathrm{s}}$, a equação (1) será considerada em estado estacionário. Neste caso as seguintes igualdades, $y(k)=y(k-1)=$ $\ldots=y\left(k-n_{y}\right)=\bar{y}$ e $u(k-1)=u(k-2) \ldots=$ $u\left(k-n_{u}\right)=\bar{u}$ podem ser utilizadas. Isso é o mesmo que aplicar o teorema do valor final para o modelo representado pela equação (1), linearizado em torno de uma entrada em degrau de amplitude final $\bar{u}$. Usando a definição de coeficientes de agrupamentos, o modelo NARX polinomial pode ser escrito na forma

$$
\begin{aligned}
\bar{y}=\Sigma_{0}+\Sigma_{y} \bar{y}+\Sigma_{u} \bar{u} & +\sum_{m=1}^{\ell-1} \sum_{p=1}^{\ell-m} \Sigma_{y^{p} u^{m}} \bar{y}^{p} \bar{u}^{m} \\
& +\sum_{p=2}^{\ell} \Sigma_{y^{p}} \bar{y}^{p}+\sum_{m=2}^{\ell} \Sigma_{u^{m}} \bar{u}^{m},
\end{aligned}
$$

sendo que os termos foram separados em: termo constante, $\Sigma_{0}$; termos lineares em $y, \Sigma_{y} \bar{y}$; termos lineares em $u, \Sigma_{u} \bar{u}$; termos cruzados $\sum_{m=1}^{\ell-1} \sum_{p=1}^{\ell-m} \Sigma_{y^{p} u^{m}} \bar{y}^{p} \bar{u}^{m}$; termos não-lineares em $y, \sum_{i=1}^{\ell} \Sigma_{y^{i}} \bar{y}^{i} ;$ e termos não-lineares em $u, \sum_{i=1}^{\ell} \Sigma_{u^{i}} \bar{u}^{i}$.

Então, a partir da equação $(9)$, a razão estática $K_{\mathrm{s}}$ pode 
ser expressa por

$$
\begin{gathered}
\hat{K}_{\mathrm{S}}(\bar{u}, \bar{y})=\frac{\bar{y}}{\bar{u}}= \\
\frac{\Sigma_{0} / \bar{u}+\Sigma_{u}+\sum_{m=2}^{\ell} \Sigma_{u^{m}} \bar{u}^{m-1}}{1-\Sigma_{y}-\sum_{m=1}^{\ell-1} \sum_{p=1}^{\ell-m} \Sigma_{y^{p} u^{m}} \bar{y}^{p-1} \bar{u}^{m}-\sum_{p=2}^{\ell} \Sigma_{y^{p}} \bar{y}^{p-1}} .
\end{gathered}
$$

\subsection{Função Estática}

Como função estática de um modelo entender-se-á a função que descreve a relação entre a saída em estado estacionário $\bar{y}$ e a respectiva entrada, também em estado estacionário, $\bar{u}$. Essa função pode ser entendida também como a função de pontos fixos para um sistema não-autônomo.

Uma vez obtida a expressão para ganho estático de um modelo, equação (10), a expressão para função estática pode ser determinada por

$$
\bar{y}=\hat{K}_{\mathrm{s}}(\bar{y}, \bar{u}) \bar{u}
$$

então, a partir da equação (10)

$$
\bar{y}=\frac{\Sigma_{0}+\Sigma_{u} \bar{u}+\sum_{m=2}^{\ell} \Sigma_{u^{m}} \bar{u}^{m}}{1-\Sigma_{y}-\sum_{m=1}^{\ell-1} \sum_{p=1}^{\ell-m} \Sigma_{y^{p} u^{m}} \bar{y}^{p-1} \bar{u}^{m}-\sum_{p=2}^{\ell} \Sigma_{y^{p}} \bar{y}^{p-1}} .
$$

Se $p>2$ então não é possível se escrever (12) na forma $\bar{y}=g(\bar{u})$. Uma alternativa para contornar tal limitação é através da multiplicação de ambos os membros da equação (12) pelo denominador do segundo membro, movendo-se todos os termos para o primeiro membro. Desta forma, a saída em estado estacionário de um modelo NARX polinomial com grau de não-linearidade $\ell$ é determinada pelas raízes do seguinte polinômio:

$$
\begin{gathered}
\left(\Sigma_{y^{\ell}}\right) \bar{y}^{\ell}+\left(\sum_{i=0}^{i=1} \Sigma_{y^{\ell-1} u^{i}} \bar{u}^{i}\right) \bar{y}^{\ell-1}+\cdots+ \\
\left(\sum_{i=0}^{i=\ell-2} \Sigma_{y^{2} u^{i}} \bar{u}^{i}\right) \bar{y}^{2}+\left(1-\sum_{i=0}^{i=\ell-1} \Sigma_{y u^{i}} \bar{u}^{i}\right) \bar{y} \\
+\sum_{i=0}^{i=\ell} \Sigma_{u^{i}} \bar{u}^{i}=0
\end{gathered}
$$

podendo-se verificar que, se $\Sigma_{y^{\ell}} \neq 0$ então o modelo terá $\ell$ saídas estacionárias para cada valor de entrada $\bar{u}$. Em outras palavras, pode-se dizer que o número de saídas estacionárias possíveis de um modelo NARX polinomial é igual ao maior expoente que aparece nos termos em $y$, inclusive os termos cruzados $\Omega_{y^{p}} u^{m}$.

\section{USO DE INFORMAÇÃO AUXILIAR EM MODELOS NARX POLINOMIAIS}

As equações (5), (10) e (13) formam a base matemática necessária para uso de informação auxiliar em modelos NARX polinomiais. A informação auxiliar que se pretende utilizar são dados em estado estacionário do sistema a ser identificado. Tais dados serão utilizados tanto na seleção de estrutura quanto na estimação de parâmetros.

No caso da seleção de estrutura, o objetivo é restringir o espaço de busca da seleção de estrutura a determinados agrupamentos de termos, baseando-se em algumas características que se deseja que o modelo identificado tenha. Isso é possível em função do estudo apresentado a respeito da influência dos agrupamentos nas características estáticas do modelo.

Na estimação de parâmetros, o principal problema que se pretende atacar ocorre quando estão disponíveis dados de entrada e saída, tanto dinâmicos quanto estáticos, de um determinado sistema. O que se pretende é garantir que um modelo obtido a partir de dados dinâmicos tenha as características estáticas do sistema, mesmo quando os dados dinâmicos se encontram em uma faixa de operação mais estreita que os dados estáticos.

Esta é uma situação bastante comum. Realizar testes para coleta de dados em estado estacionário, ou mesmo conhecer a curva estática de antemão é muito mais fácil do que ter dados dinâmicos que excursionem o sistema em uma ampla faixa de operação (Johansen, 1996; Aguirre et al., 2000). A solução proposta para este problema é também aplicada para garantir que o sistema possua pontos fixos previamente determinados.

\subsection{Seleção de Estrutura}

Informação auxiliar de (i) ganho em estado estacionário, (ii) número de estados estacionários na saída do sistema e, (iii) características qualitativas a respeito do comportamento dinâmico do sistema podem exercer um papel fundamental na seleção da estrutura de modelos NARX polinomiais.

Suponha que o número de estados estacionários da saída de um sistema seja conhecido. Sendo $n$ este número, 
então deve-se excluir do conjunto de termos candidatos os agrupamentos $\Omega_{y^{p}}$ para $p>n$.

Se os autovalores do sistema variam com o ponto de operação, ou se o sistema for bilinear, então deve-se selecionar agrupamentos com termos $\Omega_{y^{p} u^{m}} \operatorname{com} p \neq 0$ e $m \neq 0$.

Quando dados de entrada e saída em estado estacionário estiverem disponíveis, então por meio de um ajuste de curva polinomial pode ser determinado o menor grau, $m$, do polinômio que melhor se ajusta aos dados. Desta forma seleciona-se os agrupamentos de termos que devem pertencer ao conjunto de termos candidatos.

\subsection{Estimação de Parâmetros}

Na estimação de parâmentros de modelos NARMAX polinomiais, dados estáticos de entrada e saída são de fundamental importância. A utilização desta informação se baseia na equação (12).

O método utilizado consiste em, a partir da estrutura selecionada, escrever a função que relaciona $\bar{y}=f(\bar{u})$ conforme equação (12). Neste caso $\bar{y}=f(\bar{u})$ será expressa como um polinômio em $\bar{u}$ na forma

$$
\bar{y}=a_{0}+a_{1} \bar{u}+a_{2} \bar{u}^{2}+\ldots+a_{\ell} \bar{u}^{\ell},
$$

sendo $a_{0,1,2, \ldots, \ell}$ constantes que podem ser expressas como função dos coeficientes de agrupamentos. A partir da equação (14) e dos dados estáticos, pode-se ajustar um polinômio usando-se qualquer método de otimização para estimar os coeficientes $a_{0,1,2, \ldots, \ell}$. Com estes coeficientes, equações que relacionam os coeficientes aos agrupamentos de termos são determinadas. Tais equações são utilizadas como restrições na estimação de parâmetros. Neste caso podem ser utilizados métodos de otimização linear e não-linear com restrições (Corrêa, 2001; Barroso, 2001).

\section{RESULTADOS NUMÉRICOS}

Nesta seção serão apresentados resultados práticos da aplicação da identificação caixa-cinza utilizando modelos NARX polinomiais. Dois sistemas serão utilizados, um sistema simulado e outro real.

A identificação caixa-cinza aqui apresentada consiste na utilização de informação auxiliar de ganho em estado estacionário durante a estimação de parâmentros de modelos NARMAX polinomiais. Para estimar parâmetros com restrições será utilizado o algoritmo elipsóide (AE) de programação não-linear (Dziuban et al., 1985; Kim et al., 1994). Os resultados obtidos são comparados com o Estimador Estendido de Mínimos Quadrados (EMQ) (Aguirre, 2000a), ou seja, modelos obtidos via identificação caixa-cinza são comparados com modelos caixapreta.

\subsection{Sistema Simulado}

O sistema hipotético simulado, é um sistema de primeira ordem representado por (Haber e Unbehauen, 1990):

$$
[1+\alpha u(t)] \frac{d y}{d t}+y(t)-u(t)=0
$$

ou seja, trata-se de um sistema cujo ganho é constante e a constante de tempo varia com o ponto de operação na seguinte forma $\tau(u)=1+\alpha u(t)$. A Equação (15) foi simulada com $\alpha=0,5$, gerando conjuntos de dados distintos para identificação e para validação.

A partir da massa de dados gerada para identificação, deu-se origem a mais duas massas de dados, sendo que nestas duas últimas foi adicionado ruído, simulando erro de medição. Então, para o processo de estimação de parâmetros será usado: (i) Massa de dados dad_ctv1 - sinal original $y(k)$, sem ruído; (ii) Massa de dados dad_ctv2 sinal com ruído adicionado $y_{2}(k)=y(k)+\epsilon$, sendo que $\epsilon$ possui distribuição gaussiana de média zero e variância $\sigma^{2}=0,01$; (iii) Massa de dados dad_ctv3 - sinal com ruído adicionado $y_{3}(k)=y(k)+\epsilon$, sendo que $\epsilon$ possui distribuição gaussiana de média zero e variância $\sigma^{2}=0,05$. Trechos dos sinais usados na identificação estão apresentados na Figura 7. Como pode ser observado, a entrada possui amplitude aleatória, excursionando o sistema em toda a sua faixa de operação com duração de cada patamar também aleatório. O tempo de amostragem usado foi de $T_{\mathrm{s}}=0,1 \mathrm{~s}$ e a massa de dados total possui 2002 observações.

Considerou-se que a única informação auxiliar disponível é o fato do ganho ser constante, ou seja, não varia com o ponto de operação. Esse tipo de informação auxiliar pode ser obtido junto ao pessoal de processo em muitos casos práticos. Por outro lado, a "constante de tempo" do modelo depende de $u(t)$, ou seja, varia com o ponto de operação. Desta forma objetiva-se observar o efeito sobre a aproximação da constante de tempo do sistema, tendo usado a informação de ganho para estimar os parâmetros, assim como o efeito do ruído nos parâmetos estimados pelo AE.

Como função de custo a ser minimizada pelo AE, utilizou-e a mesma função minimizada pelo EMQ, ou seja

$$
J_{M Q}=\|\mathbf{y}-\mathbf{\Psi} \hat{\Theta}\|^{2},
$$


(a)

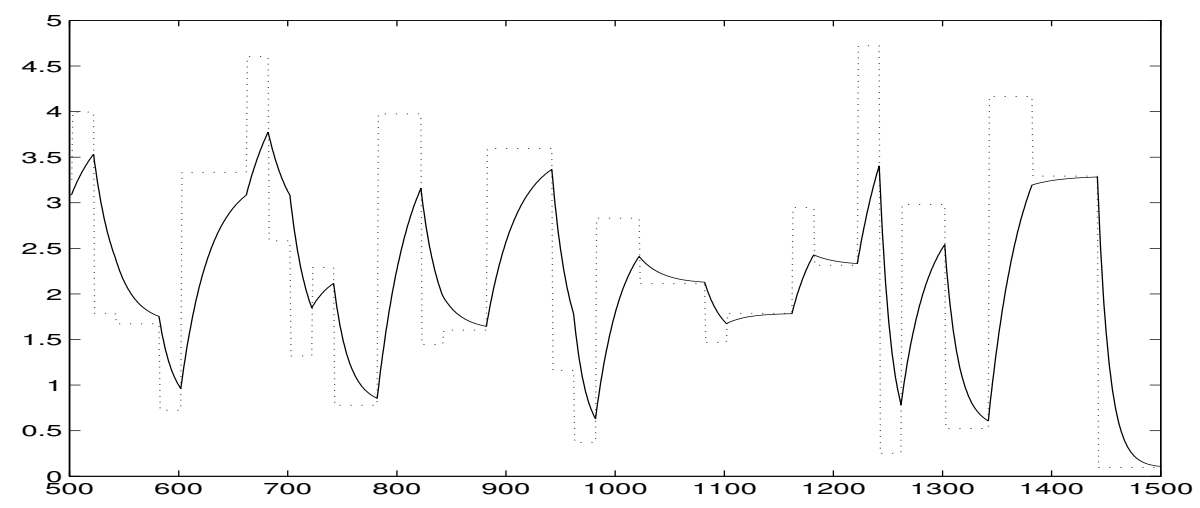

(b)

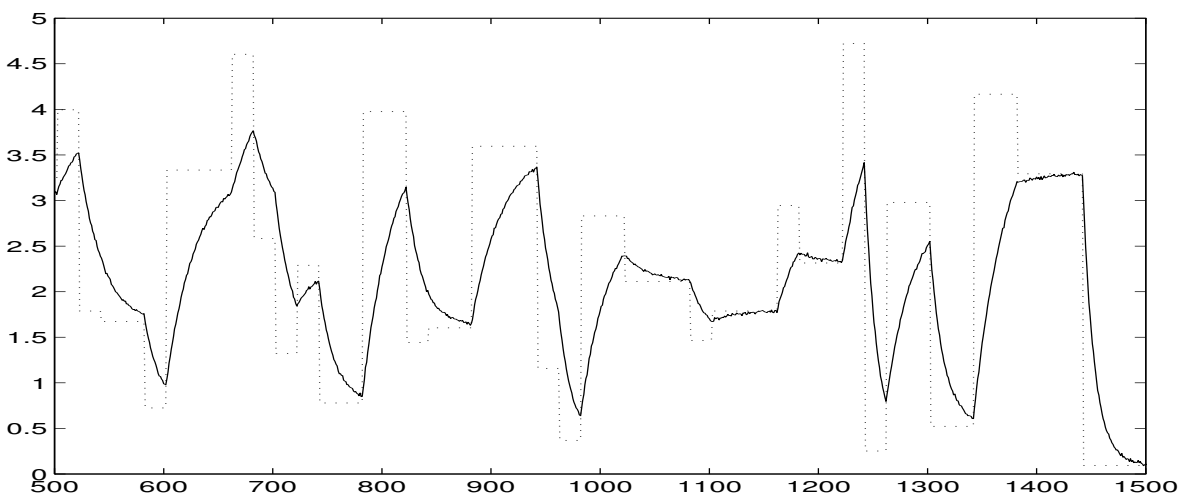

(c)

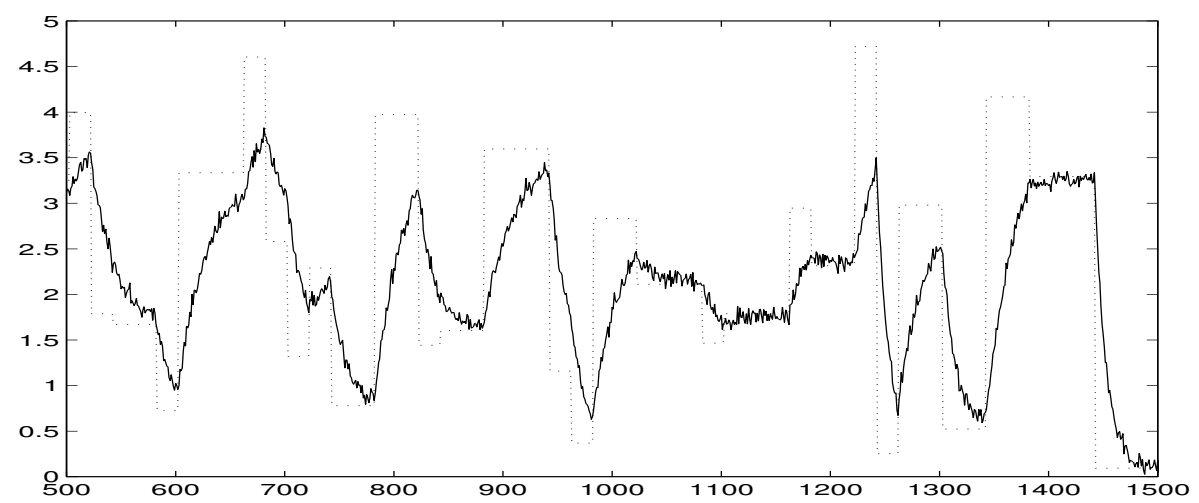

Figura 7: Parte dos dados de identificação simulados a partir da equação (15). Eixo- $x$ são as amostras e eixo-y amplitude, (-) Saída $y(k)$ e (...) entrada $u(k)$. (a) Dados dad_ctv1, (b) dados dad_ctv2 e (c) dados dad_ctv3.

sendo $\Psi$ a matriz dos regressores, contendo termos de ruído, $\hat{\Theta}$ os parâmetros estimados e y o vetor contendo os dados medidos na saída do sistema.

\subsection{Modelo NARX Polinomial}

Para este caso, não se utilizou informação auxiliar na seleção dos termos candidatos. Procurou-se obter um modelo de ordem $n_{y}=1$ e grau de não-linearidade $\ell=2$. A estrutura do modelo foi obtida pelo algoritmo ERR, obtendo o seguinte resultado:

$$
\begin{array}{r}
y(k)=\theta_{1} y(k-1)+\theta_{2} u(k-1)+\theta_{3} y(k-1) u(k-1)+ \\
\theta_{5} u(k-1)^{2}+e(k), \quad(17)
\end{array}
$$

sendo que $\Sigma_{y_{1}}=\theta_{1}, \Sigma_{u}=\theta_{2}, \Sigma_{y_{1} u}=\theta_{3}$ e $\Sigma_{u^{2}}=\theta_{5}$. Verifica-se que há apenas um termo de cada agrupamento de termos, então cada coeficiente de agrupamento corresponde a apenas um parâmetro do modelo. Observa-se também que o modelo possui apenas um estado estacionário, $\bar{y}$, para cada entrada constante, $\bar{u}$.

A partir da estrutura selecionada, é possível escrever a 
(a)

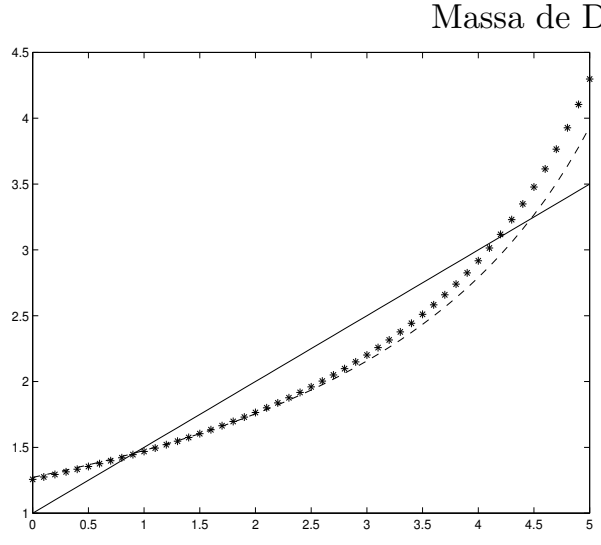

Massa de Dados dad_ctv2

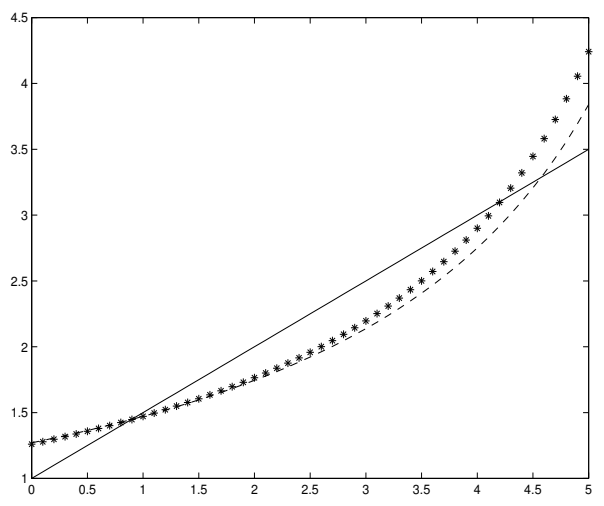

Massa de Dados dad_ctv3

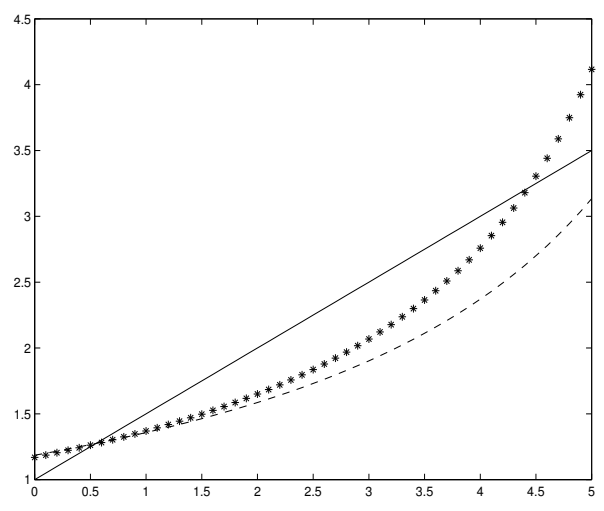

(b)
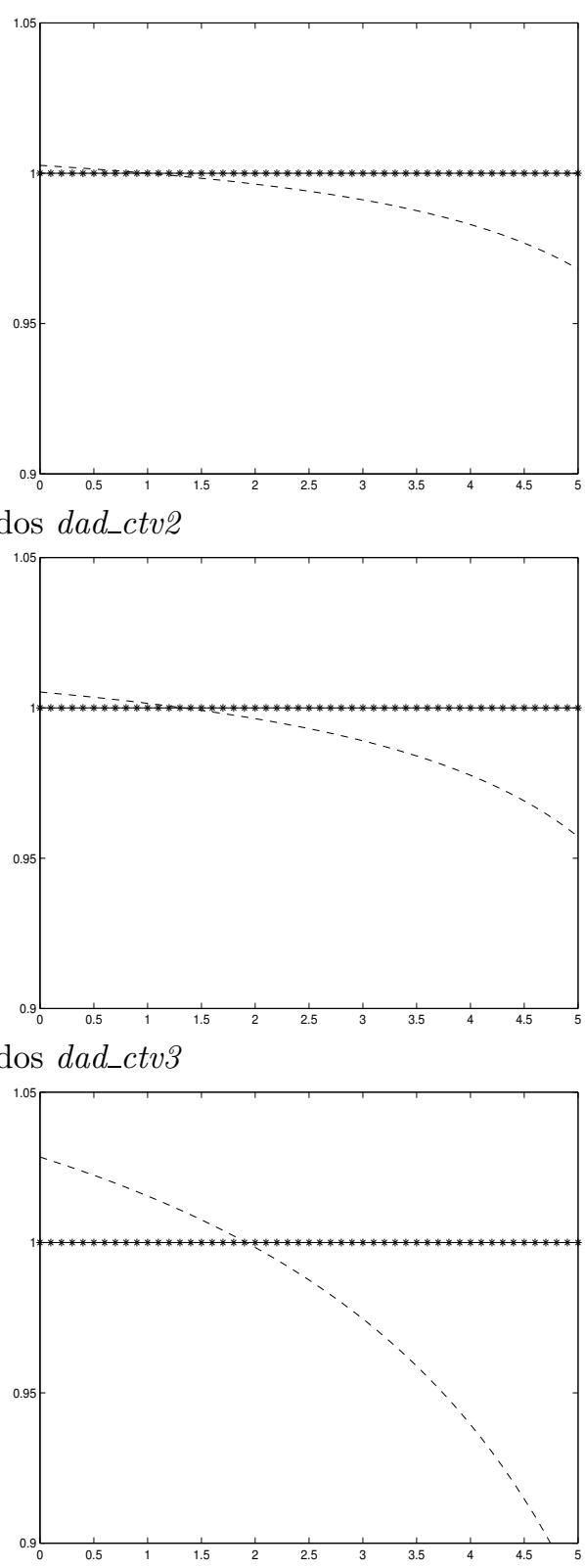

Figura 8: Funções de "constantes de tempo" e ganhos dos modelos NARX polinomiais obtidos a partir da simulação da eq. (15). Coluna (a) Curvas de "constante de tempo", eixo- $x$ é $\bar{u}$ e eixo- $y$ é $\tau$ em segundos. Coluna (b) curvas de ganho, eixo- $x$ é $\bar{u}$ e eixo- $y$ é $k(\bar{u})$. (-) Sistema simulado $(* *)$ modelos AE e (- $)$ modelos EMQ.

seguinte função que relaciona $\bar{y}=f(\bar{u})$ :

$$
\bar{y}=\frac{\theta_{2} \bar{u}+\theta_{5} \bar{u}^{2}}{\left(1-\theta_{1}\right)-\theta_{3} \bar{u}},
$$

ou ainda, o ganho em estado estacionário escrito como

$$
\frac{\bar{y}}{\bar{u}}=\frac{\theta_{2}+\theta_{5} \bar{u}}{\left(1-\theta_{1}\right)-\theta_{3} \bar{u}},
$$

não sendo usados os coeficientes de agrupamentos, pois, conforme mencionado, cada coeficiente corresponde a apenas um parâmetro no modelo.

A informação auxiliar utilizada é o ganho ser constante e igual à unidade. Então, para que o modelo possua esta característica é necessário que $\theta_{2}=\left(1-\theta_{1}\right)$ e $\theta_{5}=-\theta_{3}$. Uma vez que o $\mathrm{AE}$ não admite restrições de 
Tabela 2: Parâmetros estimados do modelo NARMAX polinomial via EMQ e EA a partir das massas de dados dad_ctv1, dad_ctv2 e dad_ctv3.

\begin{tabular}{|c|c|c|c|c|c|c|}
\hline & \multicolumn{3}{|c|}{ EMQ } & \multicolumn{3}{|c|}{ Algoritmo Elipsóide } \\
\hline & dad_ctv1 & dad_ctv2 & dad_ctv3 & $d a d \_c t v 1$ & dad_ctv2 & dad_ctv3 \\
\hline$\hat{\theta}_{1}$ & 0,9244 & 0,9243 & 0,9191 & 0,9235 & 0,9237 & 0,9180 \\
\hline$\hat{\theta}_{2}$ & 0,0758 & 0,0761 & 0,0832 & 0,0765 & 0,0763 & 0,0820 \\
\hline$\hat{\theta}_{3}$ & 0,0101 & 0,0100 & 0,0099 & 0,0107 & 0,0106 & 0,0116 \\
\hline$\hat{\theta}_{5}$ & $-0,0103$ & $-0,0103$ & $-0,0111$ & $-0,0107$ & $-0,0106$ & $-0,0116$ \\
\hline$r_{1}$ & 0,0002 & 0,0004 & 0,0023 & 0,0000 & 0,0000 & 0,0000 \\
\hline$r_{2}$ & $-0,0002$ & $-0,0003$ & $-0,0012$ & 0,0000 & 0,0000 & 0,0000 \\
\hline
\end{tabular}

igualdade, as seguintes restrições foram impostas para estimação de parâmetros

$$
\begin{aligned}
& -\epsilon<a_{2}-\left(1-a_{1}\right)<\epsilon \\
& -\epsilon<a_{5}+a_{3}<\epsilon,
\end{aligned}
$$

sendo $\epsilon=1 \times 10^{-4}$ a precisão requerida.

A Tabela 2 apresenta os valores dos parâmetros estimados via EMQ e AE, para cada massa de dados apresentada na Figura 7. As primeiras quatro linhas da tabela apresentam os valores estimados dos parâmetros da equação (17), e nas duas últimas tem-se o resultado das equações de restrições mostradas em (20).

Pode-se observar na Tabela 2 que os valores dos parâmetros estimados pelos dois métodos são próximos, com diferenças apenas na terceira casa decimal. Tal fato se justifica, pois os dois métodos minimizam a mesma função custo, só que, devido às restrições impostas ao método $\mathrm{AE}$, este garante que o modelo identificado tenha ganho em estado estacionário igual ao do sistema simulado.

Com o objetivo de se comparar os modelos obtidos pelo EMQ e AE, utilizou-se a equação que fornece a "constante de tempo" de cada modelo em função de $u(t)$. Considerando a estrutura da equação (17) a "constante de tempo" é dada por

$$
\tau(\bar{u})=\frac{-T_{\mathrm{s}}}{\ln \left(\theta_{1}+\theta_{3} \bar{u}\right)},
$$

sendo $T_{\mathrm{s}}$ o tempo de amostragem.

A Figura 8 apresenta graficamente as funções de ganho e "constante de tempo" dos modelos com parâmetros estimados via EMQ e AE, assim como para o sistema simulado. Observa-se que a curva de ganho obtida com os parâmetros estimados pelo $\mathrm{AE}$ se sobrepõe à curva do sistema simulado, fato que não ocorre para o caso do EMQ. No gráfico da "constante de tempo" em função

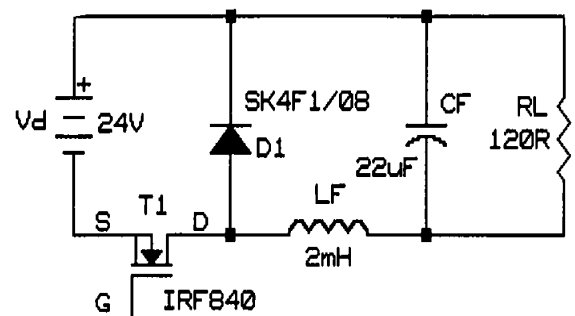

Figura 9: Conversor DC-DC Buck

do ponto de operação, observa-se que para as massas de dados dad_ctv1 e dad_ctv2 não há diferenças significativas entre os modelos. Já para a massa de dados dad_ctv3, verifica-se que os parâmetros estimados pelo AE resultaram em uma maior aproximação da curva de constante de tempo. Nota-se também que o ruído adicionado aos dados produziu efeitos similares nos dois métodos de estimação de parâmetros, embora não exista no $\mathrm{AE}$ nenhum tratamento especial à existência de ruído nos dados, ou seja, o AE estima os parâmetros de uma estrutura NARX e não NARMAX, como é o caso do estimador EMQ.

\subsection{Sistema Real: O Conversor Buck}

O conversor Buck usado neste trabalho está apresentado na Figura 9. O sistema e os dados de identificação utilizados são os mesmo considerados em (Aguirre et al., 2000). Durante todo o teste a tensão DC da fonte, $V_{d}$, é mantida constante em $24 \mathrm{~V}$. O MOSFET IRF840 é chaveado pela atuação na sua porta G. O ciclo de trabalho, definido pela proporção do tempo ligado e o período total, $D=T_{\text {on }} / T$, é variado usando a Modulação por Largura de Pulso (PWM, do inglês Pulse 


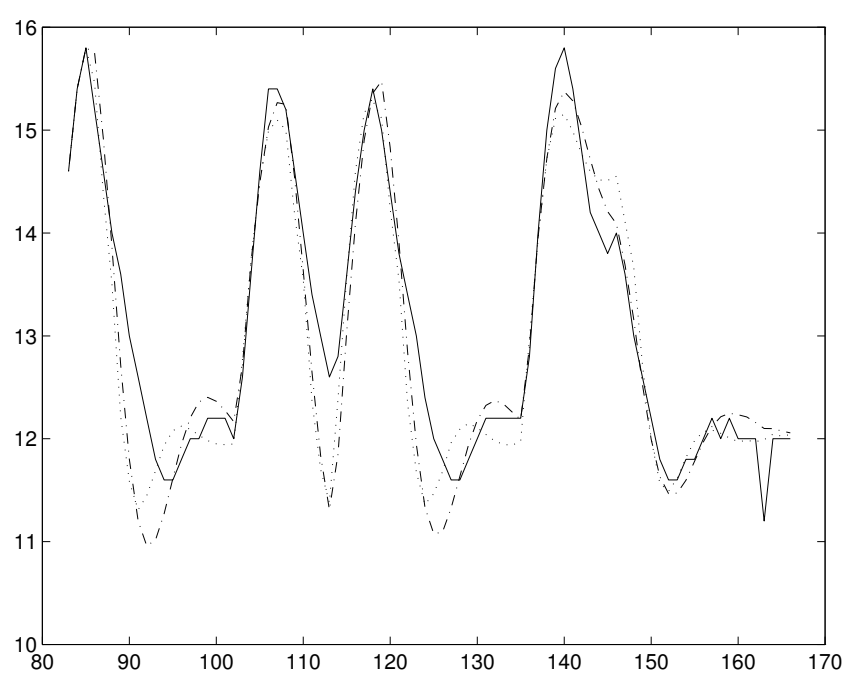

Figura 10: Predição livre dos modelos e do conversor Buck. Eixo- $x$ são amostras e eixo- $y$ são: (linha) saída do sistema, (traço-ponto) modelo EMQ e (pontilhado) modelo EA.

Width Modulation) a uma freqüência de chaveamento de $1 / T=33 \mathrm{kHz}$. Esta modulação é realizada pelo circuito integrado LM3524.

A freqüência de chaveamento utilizada resulta em um modo de operação contínuo. Quando o ciclo de trabalho tende à unidade, a corrente flui através de LF e a tensão de saída $V_{o}$ aumenta, pois $V_{d}$ é aplicada em série com LF e CF. Por outro lado, quando $D$ se aproxima de zero, a tensão da saída diminui de acordo com o regime dinâmico definido por CF, LF e a carga RL.

\subsubsection{Modelagem do Conversor Buck}

O conversor Buck apresentado possui a seguinte relação de tensão em estado estacionário $V_{\mathrm{o}}=D V_{d}$. Em função da forma com que o circuito foi implementado, a relação em estado estacionário torna-se:

$$
\begin{aligned}
V_{\mathrm{o}} & =\left(1-D^{\prime}\right) V_{d} \\
& =\left(1-\frac{\bar{u}-1}{3}\right) V_{d} \\
& =\frac{4 V_{d}}{3}-\frac{V_{d}}{3} \bar{u},
\end{aligned}
$$

sendo $V_{o}$ a tensão na carga, $D^{\prime}=T_{\text {desligado }} / T$ o ciclo de trabalho, $V_{d}=24 \mathrm{~V}$ a tensão constante fornecida pela fonte e $\bar{u}=1+3 T_{\text {desligado }} / T$ é o valor de estado estacionário do sinal de controle $u(k)$. Os dados de identificação podem ser vistos na Figura 10. Pode-se observar que o sistema é excitado pelos dados somente para valores de entrada entre $u(k)=2,2 \mathrm{~V}$ a $2,5 \mathrm{~V}$. O objetivo da modelagem é obter um modelo que se ajusta à característica estática do sistema fora da região dos dados de identificação. O desempenho deste será comparado ao modelo apresentado em (Aguirre et al., 2000).

Os termos candidatos foram selecionados a partir de informação auxiliar, que neste caso é também informação a priori, pois a relação (22) é válida em geral para conversores deste tipo. Detalhes da escolha dos termos candidatos, a partir de informação auxiliar podem ser vistos em (Aguirre et al., 2000). A estrutura foi selecionada através do algoritmo ERR. Em (Aguirre et al., 2000) podem ser vistos vários modelos para esse sistema. O que melhor reproduziu a característica estática conhecida do sistema foi o modelo obtido através do uso de informação auxiliar na escolha de termos candidatos, cuja estrutura está apresentada a seguir:

$$
\begin{gathered}
y(k)=c_{1,0}(1) y(k-1)+c_{1,0}(2) y(k-2)+c_{0}+ \\
c_{0,3}(1) u(k-1)^{3}+c_{1,0}(3) y(k-3)+ \\
c_{0,3} 1,1,3 u(k-1)^{2} u(k-3)+c_{0,2}(3) u(k-3)^{3}+ \\
c_{0,2}(1,3) u(k-1) u(k-3)+c_{0,3}(1,3,3) u(k-1) u(k-3)^{2} .
\end{gathered}
$$

Como pode ser observado pela equação (23), na estrutura do modelo não aparecem os agrupamentos de termos $\Omega_{y^{p}} \operatorname{com} p>1$, o que significa que o modelo possui, para cada entrada $\bar{u}$, apenas um estado estacionário na saída. A partir da estrutura apresentada, a relação estática $\bar{y}=f(\bar{u})$ pode ser expressa por

$$
\bar{y}=\alpha_{3} \bar{u}^{3}+\alpha_{2} \bar{u}^{2}+\alpha_{0} .
$$

Usando-se o conjunto de dados estáticos obtidos pela equação (22) e estimando-se os parâmetros pelo método dos mínimos quadrados, os valores ótimos para ajuste da curva são: $\alpha_{3}^{*}=0,4391 ; \alpha_{2}^{*}=-3,3710$ e $\alpha_{0}^{*}=26,2696$, sendo que "*" significa valores ótimos dos parâmetros.

A partir dos valores ótimos apresentados acima é possível escrever as seguintes restrições à estimação de parâmetros:

$$
\begin{aligned}
& -\epsilon<0,4391-0,4391 \Sigma_{y}-\Sigma_{u^{3}}<\epsilon \\
& -\epsilon<-3,3710+3,3710 \Sigma_{y}-\Sigma_{u^{2}}<\epsilon \\
& -\epsilon<26,2696-26,2696 \Sigma_{y}-\Sigma_{0}<\epsilon
\end{aligned}
$$

sendo $\epsilon=0,01$ a precisão estabelecida.

A Tabela 3 apresenta os parâmetros estimados pelo EMQ (Aguirre et al., 2000) e AE. Pode-se observar que os módulos $\left|\alpha_{0}^{*}-\alpha_{0}\right|,\left|\alpha_{2}^{*}-\alpha_{2}\right|$ e $\left|\alpha_{3}^{*}-\alpha_{3}\right|$ são sempre menores que $\epsilon$ para o caso $\mathrm{AE}$. 
Tabela 3: Parâmetros estimados por EMQ (Aguirre et al., 2000) e AE para o modelo do Conversor Buck

\begin{tabular}{c|c|c}
\hline & EMQ & AE \\
\hline$\hat{c}_{1,0}(1)$ & 1,2013 & 0,7315 \\
$\hat{c}_{1,0}(2)$ & $-0,2608$ & $-0,0047$ \\
$\hat{c}_{0}$ & 6,2479 & 13,7292 \\
$\hat{c}_{0,3}(3)$ & $-2,6783$ & $-0,8280$ \\
$\hat{c}_{1,0}(3)$ & $-0,2080$ & $-0,2495$ \\
$\hat{c}_{0,3}(1,1,3)$ & 8,8699 & 3,6774 \\
$\hat{c}_{0,3}(3)$ & 3,6636 & 2,0210 \\
$\hat{c}_{0,2}(1,3)$ & $-0,6162$ & $-1,7617$ \\
$\hat{c}_{0,3}(1,3,3)$ & $-9,7707$ & $-4,6409$ \\
\hline$\hat{\alpha}_{0}$ & 23,3488 & 26,2659 \\
$\hat{\alpha}_{2}$ & $-2,3029$ & $-3,3704$ \\
$\hat{\alpha}_{3}$ & 0,2037 & 0,4391 \\
\hline
\end{tabular}

O resultado da predição livre dos modelos com parâmetros estimados por EMQ e AE, usando dados de validação, podem ser vistos na Figura 10. O modelo cujos parâmetros foram estimados pelo EMQ fornece o menor erro quadrático na predição livre usando dados de validação.

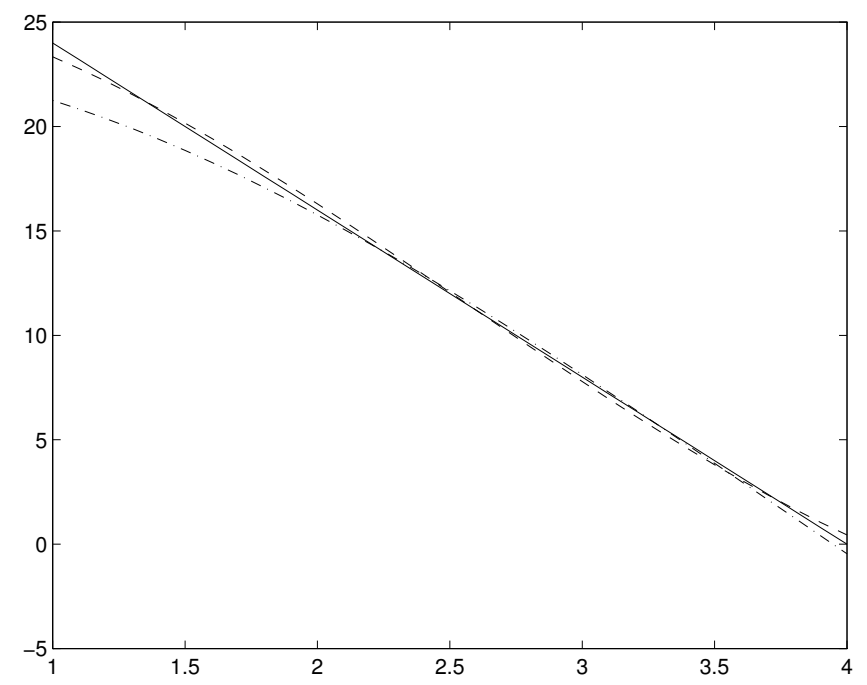

Figura 11: Função estática do (linha) sistema original, (traço-ponto) modelo EMQ e (pontilhado) modelo EA para o conversor Buck. Ambos são caixa-cinza, porém o modelo EA é mais branco que o EMQ. Eixo- $x$ é $\bar{u}$ em volts e eixo-y é $\bar{y}$ em volts. A curva do modelo EA está muito próxima à curva original.

A Figura 11 apresenta as curvas estáticas obtidas a partir dos modelo e do sistema original. Verifica-se que o $\mathrm{AE}$ fornece o melhor ajuste da curva estática fora da região dos dados de identificação. Em síntese, o uso de informação auxiliar para estimar os parâmetros possibilitou se obter um modelo que melhor reproduz a característica estática global do conversor Buck, sem uma piora significativa no desempenho dinâmico do modelo.

A melhor aproximação da característica estática significou um pior desempenho da predição livre do modelo com dados dinâmicos de identificação. De fato, um modelo obtido por identificação caixa-preta ajusta os dados de validação muito bem, mas com uma pobre característica estática e uma faixa de validade estreita (Aguirre et al., 2000). Pode-se dizer que, para o caso em questão, o preço pago pela melhor aproximação da curva estática global foi um pior desempenho no comportamento dinâmico local.

\section{COMENTÁRIOS FINAIS}

Este artigo descreveu brevemente alguns dos principais pontos em trabalhos de identificação caixa-cinza que apareceram na literatura recentemente.

Como principais pontos a favor da identificação caixacinza a literatura destaca: (i) a diminuição do número de parâmetros, (ii) maior capacidade de reproduzir características fora dos dados de identificação, (iii) maior robustez e (iv) maior adequação para o desenvolvimento de sistemas de controle. Também se identifica, nos trabalhos citados, a necessidade de se criar mecanismos mais eficientes para incorporar informações auxiliares nos modelos. O uso de informação auxiliar se dá principalmente nas etapas (i) seleção de estrutura e (ii) estimação de parâmetros. A forma com que essas informações são incorporadas dependem do tipo de representação escolhida.

Foram apresentados alguns resultados de um estudo mais aprofundado de características da representação NARMAX polinomial (Corrêa, 2001). Mostrou-se que é possível usar informação auxiliar de ganho em estado estacionário tanto na fase de seleção de estrutura quanto na estimação de parâmetros. Os resultados apresentados sugerem que, em alguns casos, o uso cuidadoso de informação auxiliar permite melhorar o desempenho dos modelos identificados.

No sistema simulado observou-se que o uso de informação de ganho em estado estacionário na estimação de parâmetros possibilitou ao modelo, além de uma melhor aproximação desta característica, uma melhor aproximação da função "constante de tempo" do sistema original. No caso real, que usou um conversor Buck, verificou-se que a técnica caixa-cinza permite ao modelo reproduzir características do sistema em regiões não visitadas pelos dados de identificação. 
Várias técnicas para identificação caixa-cinza vêm sendo propostas com o objetivo de combinar as vantagens da identificação caixa-preta e caixa-branca. Naturalmente, a identificação caixa cinza não apenas combina as vantagens, mas também apresenta algunas desvantagens das técnicas caixa-preta e caixa-branca. Em geral, a identificação caixa-cinza é mais complexa que a caixa-preta porém mais simples que a caixa-branca. A estrutura dos modelos caixa-cinza possui maior grau de liberdade que a dos modelos caixa-branca, porém menor que a dos caixa-preta. A "tonalidade de cinza" do método a ser usado dependerá do tipo de representação e da quantidade disponível de informações auxiliares, além, do objetivo de modelagem. Ressalta-se que a identificação caixa-cinza não substitui os extremos caixa-branca e caixa-preta, apenas permite, em alguns casos, conciliar as duas técnicas. A opção por qual técnica utilizar em um determinado problema prático dependerá muito do tipo de problema e da experiência do usuário. Podese dizer que mais estudos são necessários para se definir qual técnica de identificação é mais apropriada para cada situação.

\section{AGRADECIMENTOS}

Os autores agradecem ao CNPq, à FAPEMIG e ao Unileste-MG pelo suporte financeiro à pesquisa.

\section{REFERÊNCIAS}

Abony, J., Babuška, R. e Szeifert, F. (2001). Fuzzy modeling with multivariate membership functions: Gray-box identification and control design, IEEE Transactions on Systems, Man, and Cybernetics Part B 31(5): 755-767.

Aguirre, L. A. (2000a). Introdução à Identificação de Sistemas: Técnicas Lineares e Não-Lineares Aplicadas a Sistemas Reais, 2a. edição UFMG, Belo Horizonte, MG.

Aguirre, L. A. (2000b). A nonlinear dynamical approach to system identification, IEEE Circuits $\&$ Systems Society Newsletter 11(2): 10-23,47.

Aguirre, L. A. e Billings, S. A. (1995). Improved structure selection for nonlinear models based on term clustering, Int. J. Control 62: 569-587.

Aguirre, L. A., Corrêa, M. V. e Cassini, C. C. S. (2002). Nonlinearities in NARX polynomial models: representation and estimation, IEE Proceedings - Control Theory Application 9(149): 343-348.
Aguirre, L. A., Donoso-Garcia, P. F. e Santos-Filho, R. (2000). Use of a priori information in the identification of global nonlinear models - a case study using a buck converter, IEEE Trans. Circuits Syst. I 47(7): 1081-1089.

Aguirre, L. A. e Mendes, E. M. (1996). Nonlinear polynomial models: Structure, term clusters and fixed points, Int. J. Bif. Chaos 6(2): 279-294.

Aguirre, L. A., Rodrigues, G. G. e Mendes, E. M. (1997). Nonlinear identification and cluster analysis of chaotic attractors from a real implementation of Chua's circuit, Int. J. Bif. Chaos 7(6): 1411-1423.

Amaral, G. F. V. (2001). Uso de Redes Neurais e Conhecimento a Priori na Identificação de Sistemas Dinâmicos Não-Lineares, Dissertação de Mestrado, PPGEE, Universidade Federal de Minal Gerais, Belo Horizonte, Brasil.

Bai, E. W. e Sastry, S. S. (1986). Parameter identification using prior information, Int. J. Control 44(2): 455-473.

Barroso, M. F. S. (2001). Métodos de Otimização MonoObjetivo Aplicados á Identificação Caixa-Cinza de Sistemas Não-Lineares, Dissertação de Mestrado, PPGEE, Universidade Federal de Minas Gerais, Belo Horizonte, Brasil.

Billings, S. A., Chen, S. e Korenberg, M. J. (1989). Identification of MIMO nonlinear systems using a forward-regression orthogonal estimator, Int. J. Control 49(6): 2157-2189.

Bohlin, T. (1994). A case study of grey box identification, Automatica 30(2): 307-318.

Bohlin, T. e Graebe, S. T. (1995). Issues in nonlinear stochastic grey box identification, Int. J. of Adaptative Control and Signal Processing 9(6): 465.

Corrêa, M. V. (2001). Identificação Caixa-Cinza de Sistemas Não-Lineares Utilizando Representações NARMAX Racionais e Polinomiais, Tese de Doutorado, PPGEE, Universidade Federal de Minal Gerais, Belo Horizonte, Brasil. http://www.cpdee.ufmg.br/ MACSIN.

Corrêa, M. V., Aguirre, L. A. e Saldanha, R. R. (2002). Using prior knowledge to constrain parameter estimates in nonlinear system identification, IEEE Trans. Circuits Syst. I 9(49): 1376-1381.

Cubillos, F. A., Alvarez, P. I., Pinto, J. C. e Lima, E. L. (1996). Hybrid-neural modeling for particulate solid drying processes, Power Technology (87): 153160. 
Cubillos, F. A. e Lima, E. L. (1997). Identification and optimizing control of a roucher flotation circuit using an adaptable hybrid-neural model, Minerals Engineering 10(7): 707-721.

Dziuban, S. T., Ecker, J. G. e Kupferschmid, M. (1985). Using deep cuts in an ellipsoid algorithm for nonlinear programming, Mathematical Programming Study 25: 93-107.

Funkquist, J. (1997). Grey-box identification of a continuous digester - a distributed-parameter process, Control Eng. Practice 5(7): 919-913.

Garcia, C. (1997). Modelagem e simulação de processos industriais e de sistemas eletromecânicos, EDUSP, São Paulo.

Haber, R. e Unbehauen, H. (1990). Structure identification of nonlinear dynamic systems - a survey on input/output approaches, Automatica 26(4): 651677 .

Joerding, W. J. e Meador, J. L. (1991). Encoding a priori information in feedforward networks, Neural Networks 4: 847-856.

Johansen, T. A. (1996). Identification of non-linear systems using empirical data and prior knowledge - an optimization approach, Automatica 32: 337.

Jorgensen, S. B. e Hangos, K. M. (1995). Grey box modelling for control: Qualitative models as a unifying framework, Int. J. of Adaptative Control and Signal Processing 9(6): 547-562.

Kárný, M., Halousková, A. e Nedoma, P. (1995). Recursive approximation by ARX model: A tool for grey box modelling, Int. J. of Adaptative Control and Signal Processing 9(6): 525-546.

Karplus, W. (1976). The spectrum of Mathematical Modelling and System Simulation. Simulation of Systems, North-Holland Publishing Company.

Kim, S., Kim, D. e Chang, K. (1994). Using two successive subgradients in the ellipsoid method for nonlinear programming, Journal of Optimization Theory and Applications 82(3): 543-554.

Korenberg, M., Billings, S., Y.P., L. e Mclloy, P. (1988). Orthogonal parameter estimation algorithm for non-linear stochastic systems, Int. J. Control 48(1): 193-210. Louisville.

Leontaritis, I. e Billings, S. (1985). Input-output parametric models for non-linear systems part II: deterministic non-linear systems, Int. J. Control 41(2): 329-344.
Lindskog, P. e Ljung, L. (1995). Tools for semiphysical modelling, Int. J. of Adaptative Control and Signal Processing 9(6): 509.

Murankami, K. e Seborg, D. E. (2000). Constrained parameter estimation with applications to bleding operations, Journal of Process Control 10: 195-202.

Neponuceno, E. G. (2002). Identificação Multiobjetivo de Sistemas Não-Lineares, Dissertação de Mestrado, PPGEE, Universidade Federal de Minal Gerais, Belo Horizonte, Brasil.

Pearson, R. K. e Pottmann, M. (2000). Gray-box identification of block-oriented nonlinear models, Journal of Porcess Control 10: 301-315.

Petrick, M. H. e Wigdorowitz, B. (1997). A priori nonlinear model structure selection for system identification, Control Eng. Pratice 5(8): 1053-1062.

Pottmann, M. e Pearson, R. K. (1998). Block-oriented NARMAX models with output multiplicities, $A I$ ChE Journal 44(1): 131-140.

Sadegh, P., Holst, J., Madsen, H. e Melgaard, H. (1995). Experiment design for grey box identification, Int. J. of Adaptative Control and Signal Processing 9(6): 491 .

Simutis, R., Oliveira, R., Manikowski, M., Azevedo, S. F. e Lübbert (1997). How to increase the performance of models for process optimization and control, Journal of Biotechnology 59: 73-89.

Sohlberg, B. (1998). Supervision and Control for Industrial Processes - Using Grey Box Models, Predictive Control and Fault Detection Methods, SpringerVerlag.

Thompson, M. L. e Kramer, M. A. (1994). Modeling chemical processes using prior knowledge and neural networks, Automatica 40(8): 1328-1340.

Tulleken, H. J. A. F. (1993). Grey-box modelling and identification using physical knowledge and bayesian techiniques, Automatica 29(2): 285-308.

Wiener, N. (1958). Nonlinear problems in random theory, in J. Wiley e Sons (eds), The Technology Press, M.I.T. New York, NY. 